\title{
Evidence that supports policies to delay cervical screening until after age 25 years
}

\author{
James A. Dickinson MB PhD, Gina Ogilvie MD DrPH, Dirk Van Niekerk MBBCh MMed, Cathy Popadiuk MD
}

Cite as: CMAJ 2017 March 13;189:E380-1. doi: 10.1503/cmaj.160636

$\mathbf{N}$ ew guidelines for cervical screening from Alberta ${ }^{1}$ and British Columbia ${ }^{2}$ recommend increasing the starting age from 21 to 25 years. These guidelines follow the recommendations published by the Canadian Task Force on Preventative Health Care in 2013, ${ }^{3}$ and are similar to many other highincome countries. Cervical cancer mostly has a long natural history and, consequently, is uncommon in women under age 25 years. Cervical screening among this age group has minimal impact on rates of carcinoma but does lead to overdiagnosis and overtreatment of precancerous lesions that are destined to regress or not progress to cancer until later. Physicians may be concerned that delaying screening for cervical cancer will lead to reduced testing for sexually transmitted infection and too few pelvic examinations. However, delaying the onset of cervical screening for women at average risk until after age 25 years strikes the best balance between screening benefits and substantial harms. We summarize the evidence on lead time to the development of invasive cervical cancer, incidence of cervical precancerous lesions and invasive cancer in young women, and harms of screening in young women, which has informed policies to delay the age at which cervical screening begins.

Cervical cancer is caused by high-risk human papillomavirus (HPV) infection in susceptible women who do not clear it. Persistent infection may develop into precancer, and for a small proportion of patients, invasive cervical cancer. According to multiple epidemiologic studies, before screening, incidence of cervical cancer among unscreened populations was high from 35 to 65 years, which suggests a long lead-time from initial HPV infection at some time after the onset of sexual activity to the development of invasive cancer. ${ }^{4,5}$ There are few studies of the untreated natural history, but a cohort study in New Zealand found that invasive cancer developed in $30 \%$ of women with carcinoma in situ (cervical intraepithelial neoplasia 3) over 30 years of follow-up. ${ }^{6}$

Invasive cancer of the cervix is uncommon among women under age 25 years, regardless of screening practices. In Canada, the incidence among young women between 15 and 19 years of age has not changed since the era before widespread screening began in 1971: three per million. ${ }^{5}$ Among women between 20 and 24 years of age, incidence decreased from 3.2 to 1.2 per 100000 , with no change in uncommon mortality from invasive cervical

\section{KEY POINTS}

- Cervical screening guidelines now recommend starting screening after or from age 25 years.

- The natural history of progression from human papillomavirus infection to cellular abnormalities shows that most abnormalities regress, with invasive cancer developing in a few women, mostly between ages 30 to 50 years.

- Canadian epidemiology and the experience of other countries shows minimal disease in young women and that screening is ineffective in preventing those few cases but does cause substantial harms.

- Screening must balance the potential benefits of finding and treating early disease against the harms caused by overdiagnosis and treatment of early abnormalities that would regress if never found.

cancer despite programs of annual screening, a pattern that reflects what has been observed in other high-income countries. ${ }^{5}$

On the other hand, abnormal screening results and precancerous cervical lesions are commonly observed in younger women. Rates of abnormal pap tests among women 20 to 24 years of age vary from $7.3 \%$ in British Columbia ${ }^{7}$ to $23 \%$ in Newfoundland and Labrador. ${ }^{8}$ Most abnormalities are low grade and represent transient HPV infections that regress without intervention: few progress to high-grade precancer. A study in California involving 95 women under the age of 24 years with a diagnosis of cervical intraepithelial neoplasia grade 2 , which is considered a highgrade lesion, reported that lesions in two-thirds of the women had regressed by two years of follow-up, and no progression to invasive carcinoma was noted. ${ }^{9}$ Although $37 \%$ of lesions were still present, persistent lesions can be treated later without adverse physical consequence from the delay. Furthermore, with maturation of the cohorts of women who received HPV vaccination at a young age, high-grade precancerous cervical lesions and the incidence of invasive cancer are expected to be reduced further.

Therefore, screening young women leads to substantial identification and overtreatment of transient changes, which could potentially cause serious harms. A study in the United Kingdom estimated that to prevent one invasive cancer in women who are 20 to 24 years old would require between 12500 and 40000 additional 
screening tests and between 300 and 900 excisional procedures (e.g., loop electrosurgical excision procedure or cone biopsy). ${ }^{10}$ Furthermore, a systematic review and meta-analysis that assessed the effect of treatment for cervical intraepithelial neoplasia on obstetric outcomes ${ }^{11}$ showed that cervical excisional procedures may increase the rate of preterm birth by about $5 \%$, depending on the depth of excision, and raise the risk of perinatal death by about $0.2 \%$ in each subsequent pregnancy, yet the cumulative risk of cancer developing before age 30 years is less than $0.05 \%$. In addition, diagnosis of cervical intraepitheleal neoplasia has substantial psychosocial impact, particularly for women with high-grade lesions. ${ }^{12}$

The new recommended thresholds for screening are not rigid. Some factors place women at higher risk of invasive cancer (e.g., if their sexual activity started early, which would result in a longer than average infection exposure window, multiple sexual partners, smoking or a term pregnancy as an adolescent). ${ }^{13}$ Physicians may consider screening young women with these risk factors earlier than age 25 years. Public and professional education on the importance of practicing safe sex and strategies for cancer prevention, including smoking cessation for this age group, should continue. Fortunately, noninvasive diagnostic options with high sensitivity are available for chlamydia and gonorrhoea using polymerase chain reaction tests performed on urine samples. Regular bimanual pelvic examinations for screening are no longer recommended for women at any age. ${ }^{14}$

By reducing unnecessary screening in young women, resources may be channeled to reach women who have missed recommended screening or are known to have lower rates of screening for cervical cancer, such as Indigenous women, women living in rural areas and some groups of immigrants. A first test for these women at higher risk provides more value than testing women at low risk who are under the age of 25 years.

\section{References}

1. Toward Optimized Practice (TOP) Working Group for Cervical Cancer. Cervical Cancer Screening: Clinical Practice Guideline. 2016. Edmonton: Toward Optimized
Practice; 2016. Available: www.topalbertadoctors.org/download/587/cervical\%20 cancer\%20guideline.pdf?_20160602171952 (accessed 2016 May 16).

2. British Columbia cervical cancer screening guidelines. Vancouver: BC Cancer Agency; 2016.

3. Canadian Task Force on Preventive Health Care, Dickinson J, Tsakonas E, Conner Gorber S, et al. Recommendations on screening for cervical cancer. CMAJ 2013;185:35-45.

4. Bosch FX, Lorincz A, Munoz N, et al. The causal relation between human papillomavirus and cervical cancer. J Clin Pathol 2002;55:244-65.

5. Dickinson JA, Stankiewicz A, Popadiuk C, et al. Reduced cervical cancer incidence and mortality in Canada: national data from 1932 to 2006. BMC Public Health 2012;12:992.

6. McCredie MR, Sharples KJ, Paul C, et al. Natural history of cervical neoplasia and risk of invasive cancer in women with cervical intraepithelial neoplasia 3: a retrospective cohort study. Lancet Oncol 2008;9:425-34.

7. Cervical Cancer Screening Program: 2014 annual report. Vancouver: BC Cancer Agency; 2015. Available: www.bccancer.bc.ca/screening/Documents/CCSP Report-AnnualReport2014.pdf (accessed 2017 Feb. 12)

8. Fewer P. Newfoundland and Labrador Cervical Screening Initiatives Program: Report on Cervical Screening in Young Women (2016). Gander (NL): Cervical Screening Initiatives Program; 2016. Available: http://westernhealth.nl.ca/ uploads/PDFs/CSI/Cervical_Screening_in_Younger_Women_2016.pdf (accessed 2017 Feb. 21).

9. Moscicki AB, Ma Y, Wibbelsman C, et al. Rate of and risks for regression of cervical intraepithelial neoplasia 2 in adolescents and young women. Obstet Gynecol 2010;116:1373-80.

10. Landy R, Birke H, Castanon A, Sasieni P. Benefits and harms of cervical screening from age 20 years compared with screening from age 25 years. $\mathrm{Br} \mathrm{J}$ Cancer 2014;110:1841-6.

11. Kyrgiou M, Athanasiou A, Paraskevaidi M, et al. Adverse obstetric outcomes after local treatment for cervical preinvasive and early invasive disease according to cone depth: systematic review and meta-analysis. BMJ 2016;354:i3633. doi: 10.1136/bmj.i3633.

12. Pirotta M, Ung L, Stein A, et al. The psychosocial burden of human papillomavirus related disease and screening interventions. Sex Transm Infect 2009;85:508-13.

13. International Collaboration of Epidemiological Studies of Cervical Cancer. Cervical carcinoma and sexual behavior: collaborative reanalysis of individual data on 15461 women with cervical carcinoma and 29164 women without cervical carcinoma from 21 epidemiological studies. Cancer Epidemiol Biomarkers Prev 2009;18:1060-9.

14. Tonelli M, Connor Gorber S, Moore A, et al. Recommendations on routine screening pelvic examination: Canadian Task Force on Preventive Health Care adoption of the American College of Physicians guideline. Can Fam Physician 2016;62:211-4.

\section{Competing interests: None declared.}

Affiliations: Family Medicine and Community Health Sciences (Dickinson), University of Calgary, Calgary, Alta; Faculty of Medicine, The University of British Columbia, and Women's Health Research Institute, BC Centre for Disease Control (Ogilvie); Department of Pathology, The University of British Columbia, and
Cervical Screening Program of the British Columbia Cancer Agency (Van Niekerk), Vancouver, BC; Department of Obstetrics and Gynecology, Faculty of Medicine, Memorial University, and Provincial Cervical Screening Initiative Program (Popadiuk), St. John's, NL

Contributors: All of the authors made substantial contributions to the conception and design of the work, revised it critically for intellectual content, approved the final version to be published and agreed to be accountable for all aspects of the work.

This article has been peer reviewed.

Correspondence to: James Dickinson, dickinsj@ucalgary.ca 